\title{
PARK-PEOPLE INTERFACE: A CASE STUDY OF AYODHYAPURI VDC, CHITWAN, NEPAL
}

\author{
Anita Pant* and Shivraj Bhatta** \\ *Central Department of Environmental Science, Tribhuvan University,Kathmandu, Nepal. \\ **World Wide Fund for Nature (WWF), Baluwatar, Kathmandu, Nepal.
}

\begin{abstract}
The study in Ayodhyapuri Village Development Committee in Buffer Zone of Chitwan National Park revealed that the main source of energy is fuel wood, most of which is supplied from Buffer Zone Community Forestry (BZCF) and Chitwan National Park (37.93\%) and partially from private land (2.15\%). Agriculture was main source of livelihood among the villagers. Fuel wood consumption per household was found to be $3516.11 \mathrm{~kg}$ per year in the study area. Landless and low-income households were found using more fuel wood from BZCF and National Park. The root problems in the study area are the maximum extraction of fuel wood and fodder from the CF and National Park, and the crop depredation by wildlife. The depredation of the crop by wildlife is one of the major barriers influencing the conservation attitude of local people. The CF is rich in biodiversity as revealed by presence of 36 species of trees, 54 species of shrubs and 66 species of herbs. Shorea robusta is the most dominating species in both trees and shrub strata whereas Imperata cylindrica is the most frequent herbaceous species.
\end{abstract}

Key words: Buffer zone; Biodiversity; Socio-economy; Vegetation; Park and people.

\section{INTRODUCTION}

Buffer zone is defined as an area in a reserve surrounding the central core zone, in which non-destructive human activities such as ecotourism, traditional (low intensity) agriculture, or extraction of renewable natural products are permitted (Groom et.al, 2006). The buffer zone is an incremental step in effort to employ participatory methods and collaborative management in biodiversity conservation, and to shift the paradigm of 'Protection of the park from the people' towards 'Protection of the park through the people' (Budhathoki, 2005). Traditionally, National Parks and Reserve management has focused on protected areas only and rarely does park authority have jurisdiction of the land outside park boundaries. However, conservation of biological diversity inside protected areas is possible only if productive forests outside protected areas are also managed sustainably (Oldfield, 1988). The buffer zone is supposed to reduce biotic pressure in core areas and to improve the socioeconomic conditions of buffer zone communities by strengthening and mobilizing community-based buffer zone institution. There is a close linkage between forestry and rural people in Nepal where people from rural area mostly depend on the forest resources to meet their fuel wood, fodder and timber need. Over $95 \%$ of the Nepali population directly depends on the forests for their need of timber and nontimber forest products (Gautam, 2006). Threats in Chitwan National Park are mainly due to excessive poverty around the park and the lack of alternatives that force local people to intrude park's resources and degrade forest in and around CNP (NTNC, 1996). Mostly five different types of Park-People conflicts are noted in CNP namely, illegal extraction of park resources by people, livestock grazing, hunting and fishing, crop raiding by wild animals and loss of human life due to wild animals (Nepal and Weber, 1993). Due to excessive depredation of crops by animals such as rhino, deer, elephant, etc., people near CNP are forced to take action against them. Wildlife resources that are scarce and diminishing fast require human intervention with three fundamental steps to reconsolidate their resilience: explore, secure and maintain. Despite its several successes in biodiversity conservation, threats to sustainable biodiversity conservation in Chitwan continue to exist in many forms and at different scales (Budhathoki, 2005).

Chitwan National Park, established in 1973, is world renowned for its unique diversity of flora and fauna. The biological richness of the park encompasses eight ecosystem types, five wetlands and three main river system habitats. The floral diversity consists of more than 600 plant species including 10 endemic species. The faunal diversity of the park consists of 56 species of mammals, 539 species of birds, 47 species of reptiles, 9 species of amphibians and 126 species of fishes. Major vegetation types are Sal forest, Tropical mixed hardwood forest (Khair-sissoo), riverine forest and grasslands. The park is especially renowned for the protection of endangered one- horned rhinoceros, tiger and Gharial crocodile. The park also secures population of endangered species of wild gaury, wild elephant, four horned antelope, striped hyena, pangolin, Gangetic dolphin, monitor lizard and python (Basnyat, 1999). The research was carried

Author for Correspondence: Anita Pant, Central Department of Environmental Science, Tribhuvan University, Kathmandu, Nepal. Email: anitapant11@gmail.com. 
out in the Ayodhyapuri Village Development Committee (V.D.C.) which is located in the buffer zone. The research aimed to determine socio-economic status of the local people and its impact in forest resource utilization as well as to document the vegetation of buffer zone community forest.

\section{MATERIALS AND METHODS}

\section{Vegetation Survey:}

In order to design the survey, the maps of the forest patches were prepared and systematic random points were generated within the patches at an interval of $500 \mathrm{~m}$ using GIS. The latitude and longitude of these random points were noted with the help of GPS, the points were located in the field. Those points which are found to be inside the buffer zone community forest were selected to conduct the vegetation survey. Vegetation analysis carried out at 25 locations. The number of plots surveyed for trees, shrubs and herbs were 25, 50 and 50 respectively. Survey represents all three buffer zone community forests of Ayodhyapuri village.

\section{Plot Design:}

At each sampling points, altogether five plots were laid out. Quadrate plot of 20 m x 20 m were laid to study tree species. Within the tree plot, nested plot of $5 \mathrm{~m} \mathrm{x} 5 \mathrm{~m}$ were laid on the opposite corners to study shrub. Similarly, for herb species $1 \mathrm{~m}$ x $1 \mathrm{~m}$ plots nested in shrub plots were laid. All tree species having diameter at breast height (dbh) greater than $10 \mathrm{~cm}$ were taken into account within $20 \mathrm{~m}$ x $20 \mathrm{~m}$ plot. Dbh and height of all trees were measured with the help of dbh tape and clinometers, respectively. Density, relative density, frequency, relative frequency, basal area, relative basal area, importance value index, diversity index, species richness index, dominance and similarity index were also calculated using Zobel et. al (1987). The stand size classification is presented in Table 1, based on standards of the Forest Inventory Division (FSRC, 1995).

Table 1: Stand size classification

\begin{tabular}{lll}
\hline Symbol & Stand Size & Dbh $(\mathbf{c m})$ \\
\hline 1 & Sapling & $<12.5$ \\
2 & Poles & $12.5-25$ \\
3 & Small saw timber & $25-50$ \\
4 & Large saw timber & $>50$ \\
\hline
\end{tabular}

\section{Socio-economic Survey:}

The sample size (n) of the household in the study area was determined by using statistical formula given below (Arkin and Colton, 1963; cited in Sharma, 2000); at 95\% confidence level.

$$
n=\frac{N Z^{2}}{N d^{2}+Z^{2} P(1-P)}
$$

Where, $\mathrm{n}=$ sample size, $\mathrm{N}=$ total number of households, $\mathrm{Z}=$ confidence level (at 95\% level $\mathrm{z}=1.96$ ), $\mathrm{P}=$ =stimated population proportion (0.05, this maximize the sample size), $\mathrm{d}=$ =rror limit of $5 \%(0.05)$

Set questionnaires were developed to collect data related on household information, buffer zone community forest and buffer zone management issues and rhino/ wildlife related issues. The survey was conducted by direct interview with household member using structured and semi structured questionnaire with some close ended and some open-ended questions.

\section{Data Analysis:}

The quantitative data obtained from the field was analyzed using Statistical Package for Social Sciences (SPSS) and Microsoft Excel.

\section{RESULTS AND DISCUSSION}

\section{Socio-economic Survey:}

Most of the population was predominately students (35.6\%). About $16.2 \%$ of the total population was engaged in both agriculture and household work followed by $15.4 \%$ in agriculture as their main occupation. Only $2.6 \%$ of total population work in government service, $0.9 \%$ had small business along with agriculture and $9.7 \%$ went to foreign for earnings. Households were predominantly farmers. The percentage of population working in Government service is 2.6 which is less as estimated by DNPWC/PPP (2000). The population involved in the business $0.9 \%$ is comparatively much lower than given by DNPWC/PPP, 2000 at VDC level (5.7\%). On the other hand, population going outside the country for job has significantly increased as compared to report of DNPWC/PPP (2000), at that time nobody had gone outside the country for remittance.

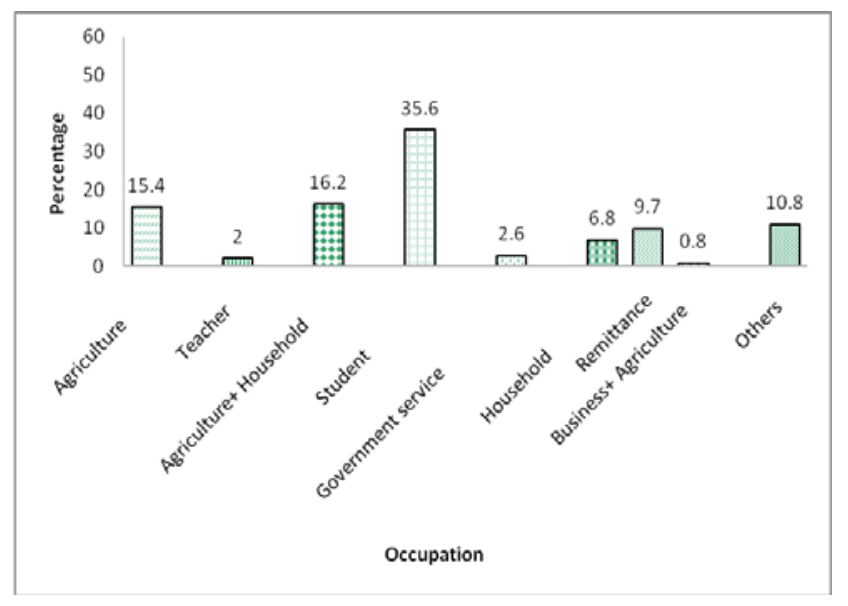

Fig. 1: Distribution of population by occupation.

The study shows that most dominant population is Brahmin and Chettri living as joint family (35.21\%) followed by dalits (16.90\%). The percentage of population living in joint family (66.19\%) was higher than those living in a nuclear 
family (Table 2). Likewise, majority of the Brahmin/Chettri were found to live in a joint family than other ethnic groups. Table 2: Family structure based on ethnicity in Ayodhyapuri VDC.

\begin{tabular}{lccc}
\hline Caste & Family structure & Total \\
\hline & Nuclear & Joint & \\
Brahmin/ Chettri & $12(16.90)$ & $25(35.21)$ & $37(52.11)$ \\
Gurung/Magar/Tamang & $1(1.40)$ & $8(11.26)$ & $9(12.67)$ \\
Newar & $1(1.40)$ & $1(1.40)$ & $2(2.82)$ \\
Tharu & - & $1(1.40)$ & $1(1.40)$ \\
Dalit & $9(12.67)$ & $12(16.90)$ & $21(29.57)$ \\
Bote & - & $1(1.40)$ & $1(1.40)$ \\
Total & $\mathbf{2 4 ( 3 3 . 8 )}$ & $\mathbf{4 7 ( 6 6 . 1 9 )}$ & $\mathbf{7 1 ( 1 0 0 )}$ \\
\hline
\end{tabular}

Data in parenthesis are in percent.

Table 3 shows the distribution of households by farm size. The population of landless households were 5.63\%, which is higher when compared with report of DNPWC/PPP (2000) (3.9\%), indicating the number of landless has increased in the area. Similarly, the population having 10-20 katta (1 katta $=0.034 \mathrm{ha}$ ) were $46.48 \%$ and that having greater than four bigha (1 bigha $=0.68 \mathrm{ha}$ ) were $2.81 \%$, has increased than that given by DNPWC/PPP (2000) 34.1\% and 1.6\%, respectively.

Table 3: Distribution of households by farm size.

\begin{tabular}{lccc}
\hline Categories & Scale & Scale in ha & Percentage of $\mathbf{H H}$ \\
\hline Landless & 0 katta & 0 & 5.63 \\
Small farm & $0-10$ katta & $0-0.34$ & 29.58 \\
Medium farm & $10-20$ katta & $0.34-0.68$ & 46.48 \\
Big farm & $1-4$ bigha & $0.68-2.72$ & 15.49 \\
Very big farm & $>4$ bigha & $>2.72$ & 2.81 \\
\hline
\end{tabular}

\section{Fuel wood Consumption and Sources:}

Buffer zone community forest, National Park and personal land were the main sources of households' fuel wood. The annual consumption of fuel wood of the sampled household was found to be $249,644 \mathrm{~kg}$. Of this, buffer zone community forest fulfilled most of the demand (59.92\%) followed by National Park (37.93\%). According to DNPWC/ PPP (2000), 44.7\% of fuel wood was supplied by National Park but the present study shows that the collection has decreased to $37.93 \%$ indicating that there is less pressure on park compared to past. Previous reports showed no fuel wood was supplied from private land (DNPWC/PPP, 2000) whereas present research showed that $2.15 \%$ is supplied by private land.

In average, fuel wood consumption per household was found to be $3516.11 \mathrm{~kg}$ per year in the study area. The per capita fuel wood consumption was found high among the landless (1051.62 kg/person/yr) and small farm households (883.64 kg/person/yr) (Table 4). The study shows that the consumption of fuel wood was related with the family size. This is because landless and small farm households were more dependent on fuel wood as they have less access to biogas, kerosene and other sources of energy.

The average fodder consumption in study area was estimated to be $895.52 \mathrm{~kg}$ per year per livestock unit (LSU) i.e $2.45 \mathrm{~kg} /$ day/LSU. It was observed that fodder consumption increased with increase in LSU except for medium farm (Figure 2). The very big farm households had higher consumption of fodder than small farm size households. Very big farm had highest value of daily fodder consumption i.e $4.01 \mathrm{~kg} /$ day/ LSU. Households with large farm required high amount of fodder as they had comparatively large number of livestock than the small farm households.

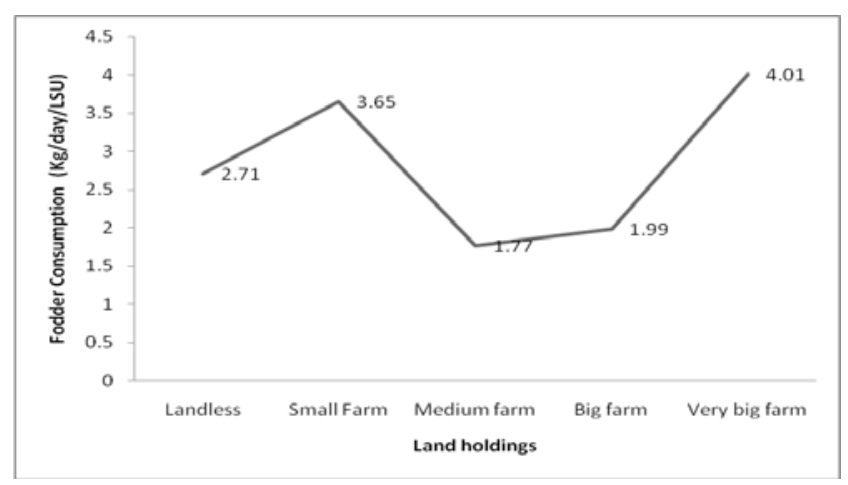

Fig. 2: Fodder consumption (kg/day/LSU) in Ayodhyapuri VDC

Table 4: Per capita fuelwood consumption (FWC) per household in Ayodhyapuri VDC.

\begin{tabular}{llclcc}
\hline Land Category & & Total HH & Total Family Size FWC (Kg/yr) & FWC(Kg/HH/yr) FWC(Kg/person/yr) \\
\hline Landless & 4 & 26 & 27342 & 6835.5 & 1051.62 \\
Small farm & 21 & 97 & 85713 & 4081.57 & 883.64 \\
Medium farm & 33 & 142 & 96824 & 2934.06 & 681.86 \\
Big farm & 11 & 74 & 35029 & 3184.45 & 473.36 \\
Very Big farm & 2 & 12 & 4736 & 2368.00 & 394.66 \\
Total & $\mathbf{7 1}$ & $\mathbf{3 5 1}$ & $\mathbf{2 4 9 6 4 4}$ & $\mathbf{3 5 1 6 . 1 1}$ & $\mathbf{7 1 1 . 2 4}$ \\
\hline
\end{tabular}


Impacts of Wild Animals on Local Community:

The entrance of rhino, elephant and wild boar was reported frequently in the field. Other wild animals such as tiger, deer, bear, monkey, fox, peacock, etc. were also noted to occur. Local people residing in the vicinity of buffer zone forest and core areas were adversely affected due to livestock and crop depredation.

There was higher pressure on crop raiding by wildlife. Of total respondents, $78.87 \%$ agreed that wildlife damage their crops and only $5.63 \%$ did not have damage to their crops because either they did not have agricultural land or protected it well by guarding the crop. The crop damage was not merely by feeding but also due to trampling during grazing crops. Rhino preferred to feed on mature paddy and wheat, and usually come solitarily in the field but sometimes accompanied by their calves. On the other hand, deer mostly fed on young plants of paddy, maize, wheat, oil seeds and potatoes.
Thus, they have considerable damage to crops. Many respondents said that they had given up growing wheat because of its heavy destruction, mainly by rhino. Feeding on the agricultural crop by wild animals generally occurred from eight in the night to dawn. Problems made by them as quoted by respondents are shown in Table 5 .

Table 5: Problems created by wild animals.

\begin{tabular}{llc}
\hline Problems by Wild Animals & No. of HH & Percentage \\
\hline Crop Damage & 56 & 78.87 \\
Crop Damage+ Livestock Loss & 8 & 11.26 \\
Livestock Loss+ Human Injury & 1 & 1.41 \\
Human Injury/Loss & 2 & 2.82 \\
None & 4 & 5.63 \\
Total & $\mathbf{7 1}$ & $\mathbf{1 0 0}$ \\
\hline
\end{tabular}

Table 6: Quantitative status of tree species in the community forests of Ayodhyapuri VDC.

\begin{tabular}{|c|c|c|c|c|c|c|c|c|}
\hline S.N. & Species & D (no./ha) & RD (\%) & $\mathbf{F}(\%)$ & RF (\%) & BA (m²/ha) & RBA (\%) & IVI \\
\hline 1 & Shorea robusta & 99.00 & 41.60 & 80.00 & 17.7 & 14.63 & 54.33 & 113.62 \\
\hline 2 & Anogeissus latifolius & 24.00 & 10.08 & 52.00 & 11.5 & 2.82 & 10.47 & 32.06 \\
\hline 3 & Terminalia alata & 11.00 & 4.62 & 36.00 & 7.96 & 3.04 & 11.28 & 23.87 \\
\hline 4 & Syzygium cumini & 10.00 & 4.20 & 28.00 & 6.19 & 0.67 & 2.49 & 12.88 \\
\hline 5 & Mallotus philippensis & 11.00 & 4.62 & 16.00 & 3.54 & 0.46 & 1.71 & 9.87 \\
\hline 6 & Semecarpus anacardium & 10.00 & 4.20 & 20.00 & 4.42 & 0.27 & 1.00 & 9.63 \\
\hline 7 & Acacia catechu & 9.00 & 3.78 & 20.00 & 4.42 & 0.22 & 0.82 & 9.02 \\
\hline 8 & Schleichera oleosa & 5.00 & 2.10 & 16.00 & 3.54 & 0.66 & 2.45 & 8.09 \\
\hline 9 & Desmodium oojeinense & 5.00 & 2.10 & 16.00 & 3.54 & 0.57 & 2.12 & 7.76 \\
\hline 10 & Antidesma acidum & 4.00 & 1.68 & 8.00 & 1.77 & 1.16 & 4.31 & 7.76 \\
\hline 11 & Glochidion velutinum & 5.00 & 2.10 & 16.00 & 3.54 & 0.28 & 1.04 & 6.68 \\
\hline 12 & Bombax ceiba & 3.00 & 1.26 & 8.00 & 1.77 & 0.61 & 2.26 & 5.30 \\
\hline 13 & Lagerstroemia parviflora & 4.00 & 1.68 & 12.00 & 2.65 & 0.21 & 0.78 & 5.12 \\
\hline 14 & Dillenia pentagyna & 5.00 & 2.10 & 12.00 & 2.65 & 0.08 & 0.29 & 5.05 \\
\hline 15 & Toona ciliata & 3.00 & 1.26 & 8.00 & 1.77 & 0.38 & 1.41 & 4.44 \\
\hline 16 & Bauhinia purpurea & 3.00 & 1.26 & 12.00 & 2.65 & 0.04 & 0.15 & 4.06 \\
\hline 17 & Mitragyna parviflora & 3.00 & 1.26 & 8.00 & 1.77 & 0.09 & 0.33 & 3.36 \\
\hline 18 & Dalbergia latifolia & 2.00 & 0.84 & 8.00 & 1.77 & 0.06 & 0.22 & 2.83 \\
\hline 19 & Cassia fistula & 2.00 & 0.84 & 8.00 & 1.77 & 0.03 & 0.11 & 2.72 \\
\hline 20 & Spondias pinnata & 2.00 & 0.84 & 8.00 & 1.77 & 0.02 & 0.07 & 2.68 \\
\hline 21 & Careya arborea & 2.00 & 0.84 & 4.00 & 0.88 & 0.15 & 0.56 & 2.28 \\
\hline 22 & Bauhinia vahlii & 2.00 & 0.84 & 4.00 & 0.88 & 0.05 & 0.18 & 1.91 \\
\hline 23 & Dalbergia sissoo & 2.00 & 0.84 & 4.00 & 0.88 & 0.03 & 0.11 & 1.84 \\
\hline 24 & Leea crispa & 1.00 & 0.42 & 4.00 & 0.88 & 0.08 & 0.29 & 1.60 \\
\hline 25 & Terminalia bellirica & 1.00 & 0.42 & 4.00 & 0.88 & 0.07 & 0.26 & 1.57 \\
\hline 26 & Alstonia scholaris & 1.00 & 0.42 & 4.00 & 0.88 & 0.05 & 0.18 & 1.49 \\
\hline 27 & Litsea monopetala & 1.00 & 0.42 & 4.00 & 0.88 & 0.04 & 0.15 & 1.45 \\
\hline 28 & Ficus semicordata & 1.00 & 0.42 & 4.00 & 0.88 & 0.04 & 0.15 & 1.45 \\
\hline 29 & Duabanga grandiflora & 1.00 & 0.42 & 4.00 & 0.88 & 0.03 & 0.11 & 1.42 \\
\hline 30 & Wendlandia puberula & 1.00 & 0.42 & 4.00 & 0.88 & 0.02 & 0.07 & 1.38 \\
\hline 31 & Cleistocalyx operculatus & 1.00 & 0.42 & 4.00 & 0.88 & 0.02 & 0.08 & 1.38 \\
\hline 32 & Swida oblonga & 1.00 & 0.42 & 4.00 & 0.88 & 0.02 & 0.07 & 1.38 \\
\hline 33 & Wendlandia exserta & 1.00 & 0.42 & 4.00 & 0.88 & 0.01 & 0.04 & 1.34 \\
\hline 34 & Phyllanthus emblica & 1.00 & 0.42 & 4.00 & 0.88 & 0.01 & 0.04 & 1.34 \\
\hline 35 & Bridelia retusa & 1.00 & 0.42 & 4.00 & 0.88 & 0.01 & 0.04 & 1.34 \\
\hline
\end{tabular}

Note: $\mathbf{D}=$ Density, $\mathbf{R D}=$ Relative density, $\mathbf{F}=$ Frequency, $\mathbf{R F}=$ Relative frequency, $\mathbf{B A}=\mathbf{B a s a l}$ Area, $\mathbf{R B A}=\mathbf{R e l a t i v e ~} \mathbf{B a s a l}$ Area, IVI= Important Value Index. 
Table 7: Quantitative studies of shrub species in the community forests of Ayodhyapuri VDC.

\begin{tabular}{|c|c|c|c|c|c|}
\hline S.No. & Species & Density (no./ha) & RD (\%) & Frequency & RF (\%) \\
\hline 1 & Shorea robusta & 3584.00 & 18.15 & 46.00 & 9.96 \\
\hline 2 & Phoenix humilis & 1480.00 & 7.49 & 46.00 & 9.96 \\
\hline 3 & Leea crispa & 2720.00 & 13.77 & 36.00 & 7.79 \\
\hline 4 & Viburnum nervosum & 832.00 & 4.21 & 24.00 & 5.19 \\
\hline 5 & Curcuma aromatic & 1160.00 & 5.87 & 22.00 & 4.76 \\
\hline 6 & Eupatorium adenophorum & 640.00 & 3.24 & 16.00 & 3.46 \\
\hline 7 & Woodfordia fruticosa & 400.00 & 2.03 & 16.00 & 3.46 \\
\hline 8 & Syzygium cumini & 504.00 & 2.55 & 14.00 & 3.03 \\
\hline 9 & Dillenia pentagyna & 416.00 & 2.11 & 14.00 & 3.03 \\
\hline 10 & Colebrookea oppositifolia & 568.00 & 2.87 & 12.00 & 2.59 \\
\hline 11 & Mallotus philippensis & 296.00 & 1.49 & 12.00 & 2.59 \\
\hline 12 & Acacia catechu & 456.00 & 2.31 & 12.00 & 2.59 \\
\hline 13 & Bauhinia vahlii & 376.00 & 1.90 & 12.00 & 2.59 \\
\hline 14 & Desmodium oojeinense & 360.00 & 1.82 & 10.00 & 2.16 \\
\hline 15 & Xeromphis spinosa & 304.00 & 1.54 & 10.00 & 2.16 \\
\hline 16 & Eulaliopsis binata & 1144.00 & 5.79 & 8.00 & 1.73 \\
\hline 17 & Indigofera cylindracea & 320.00 & 1.62 & 8.00 & 1.73 \\
\hline 18 & Phyllanthus emblica & 312.00 & 1.58 & 8.00 & 1.73 \\
\hline 19 & Musa balbisiana & 256.00 & 1.29 & 8.00 & 1.73 \\
\hline 20 & Bambusa nutans & 448.00 & 2.27 & 6.00 & 1.30 \\
\hline 21 & Thalictrum chelidonii & 168.00 & 0.85 & 6.00 & 1.30 \\
\hline 22 & Dalbergia latifolia & 104.00 & 0.53 & 6.00 & 1.30 \\
\hline 23 & Litsea monopetala & 184.00 & 0.93 & 6.00 & 1.30 \\
\hline 24 & Glochidion velutinum & 136.00 & 0.69 & 6.00 & 1.30 \\
\hline 25 & Terminalia alata & 120.00 & 0.61 & 6.00 & 1.30 \\
\hline 26 & Lagerstroemia parviflora & 184.00 & 0.93 & 6.00 & 1.29 \\
\hline 27 & Desmodium confertum & 144.00 & 0.73 & 4.00 & 0.87 \\
\hline 28 & Bridelia retusa & 32.00 & 0.16 & 4.00 & 0.87 \\
\hline 29 & Buddleja asiatica & 144.00 & 0.73 & 4.00 & 0.87 \\
\hline 30 & Imperata cylindrica & 184.00 & 0.93 & 4.00 & 0.87 \\
\hline 31 & Grewia sapida & 88.00 & 0.45 & 4.00 & 0.87 \\
\hline 32 & Antidesma acidum & 88.00 & 0.45 & 4.00 & 0.87 \\
\hline 33 & Stereospermum chelonoides & 80.00 & 0.41 & 4.00 & 0.87 \\
\hline 34 & Costus speciosus & 80.00 & 0.41 & 4.00 & 0.87 \\
\hline 35 & Murraya koenigii & 208.00 & 1.05 & 4.00 & 0.87 \\
\hline 36 & Toona ciliate & 240.00 & 1.21 & 4.00 & 0.87 \\
\hline 37 & Barleria cristata & 112.00 & 0.57 & 4.00 & 0.87 \\
\hline 38 & Gaultheria hookeri & 120.00 & 0.61 & 4.00 & 0.87 \\
\hline 39 & Dalbergia sissoo & 88.00 & 0.45 & 4.00 & 0.87 \\
\hline 40 & Zizyphus xylopyrus & 72.00 & 0.36 & 4.00 & 0.87 \\
\hline 41 & Cassia tora & 112.00 & 0.57 & 4.00 & 0.87 \\
\hline 42 & Ficus semicordata & 192.00 & 0.97 & 2.00 & 0.43 \\
\hline 43 & Cleistocalyx operculatus & 24.00 & 0.12 & 2.00 & 0.43 \\
\hline 44 & Semecarpus anacardium & 16.00 & 0.08 & 2.00 & 0.43 \\
\hline 45 & Blumea balsamifera & 24.00 & 0.12 & 2.00 & 0.43 \\
\hline 46 & Bombax ceiba & 56.00 & 0.28 & 2.00 & 0.43 \\
\hline 47 & Anogeissus latifolius & 56.00 & 0.28 & 2.00 & 0.43 \\
\hline 48 & Asparagus racemosus & 16.00 & 0.08 & 2.00 & 0.43 \\
\hline 49 & Cassia fistula & 8.00 & 0.04 & 2.00 & 0.43 \\
\hline 50 & Thysanolaena maxima & 16.00 & 0.08 & 2.00 & 0.43 \\
\hline 51 & Cassine glauca & 16.00 & 0.08 & 2.00 & 0.43 \\
\hline 52 & Wendlandia exserta & 16.00 & 0.08 & 2.00 & 0.43 \\
\hline 53 & Lyonia villosa & 40.00 & 0.20 & 2.00 & 0.43 \\
\hline 54 & Anthocephalus chinensis & 8.00 & 0.04 & 2.00 & 0.43 \\
\hline
\end{tabular}

Note: $\mathrm{D}=$ Density, $\mathrm{RD}=$ Relative Density, $\mathrm{RF}=$ Relative Frequency. 
Table 8: Quantitative status of herb strata in the community forests of Ayodhyapuri VDC.

\begin{tabular}{|c|c|c|c|c|c|}
\hline \multicolumn{2}{|c|}{ S.No. Species } & \multirow{2}{*}{$\begin{array}{l}\text { D(no./ha) } \\
55800.00\end{array}$} & \multirow{2}{*}{$\begin{array}{l}\text { RD (\%) } \\
30.56\end{array}$} & \multirow{2}{*}{$\begin{array}{l}\text { Frequency } \\
42.00\end{array}$} & \multirow{2}{*}{$\begin{array}{l}\text { RF (\%) } \\
9.81\end{array}$} \\
\hline 1 & Imperata cylindrical & & & & \\
\hline 2 & Shorea robusta & 16000.00 & 8.76 & 28.00 & 6.54 \\
\hline 3 & Phoenix acaulis & 7600.00 & 4.16 & 26.00 & 6.07 \\
\hline 4 & Eulaliopsis binata & 10800.00 & 5.90 & 20.00 & 4.67 \\
\hline 5 & Phoenix humilis & 4800.00 & 2.63 & 18.00 & 4.21 \\
\hline 6 & Pogonatherum crinitum & 12800.00 & 7.01 & 16.00 & 3.74 \\
\hline 7 & Curcuma aromatica & 3800.00 & 2.08 & 14.00 & 3.27 \\
\hline 8 & Dioscorea bulbifera & 2600.00 & 1.42 & 14.00 & 3.27 \\
\hline 9 & Smilax ovalifolia & 1200.00 & 0.66 & 12.00 & 2.80 \\
\hline 10 & Monochoria vaginalis & 3400.00 & 1.86 & 12.00 & 2.80 \\
\hline 11 & Leea crispa & 1600.00 & 0.88 & 10.00 & 2.34 \\
\hline 12 & Xeromphis spinosa & 2000.00 & 1.09 & 10.00 & 2.34 \\
\hline 13 & Trachelospermum lucidum & 2600.00 & 1.42 & 10.00 & 2.34 \\
\hline 14 & Viburnum nervosum & 2000.00 & 1.09 & 10.00 & 2.34 \\
\hline 15 & Terminalia alata & 1400.00 & 0.77 & 8.00 & 1.87 \\
\hline 16 & Piper longum & 5400.00 & 2.96 & 8.00 & 1.87 \\
\hline 17 & Indigofera cylindracea & 1000.00 & 0.55 & 6.00 & 1.40 \\
\hline 18 & Swida oblonga & 600.00 & 0.33 & 6.00 & 1.40 \\
\hline 19 & Antidesma acidum & 600.00 & 0.33 & 6.00 & 1.40 \\
\hline 20 & Boehmeria sps. & 1200.00 & 0.66 & 6.00 & 1.40 \\
\hline 21 & Flemingia chappar & 1400.00 & 0.77 & 6.00 & 1.40 \\
\hline 22 & Cissampelos pareira & 1200.00 & 0.66 & 6.00 & 1.40 \\
\hline 23 & Heteropogan contortus & 2200.00 & 1.20 & 6.00 & 1.40 \\
\hline 24 & Commelina sps. & 600.00 & 0.33 & 6.00 & 1.40 \\
\hline 25 & Coccinea grandis & 600.00 & 0.33 & 6.00 & 1.40 \\
\hline 26 & Woodfordia fruticosa & 800.00 & 0.44 & 4.00 & 0.93 \\
\hline 27 & Bambusa nutans & 2800.00 & 1.53 & 4.00 & 0.93 \\
\hline 28 & Syzygium cumini & 800.00 & 0.44 & 4.00 & 0.93 \\
\hline 29 & Cyperus rotundus & 2200.00 & 1.20 & 4.00 & 0.93 \\
\hline 30 & Thalictrum chelidonii & 600.00 & 0.33 & 4.00 & 0.93 \\
\hline 31 & Blumeopsis flava & 4800.00 & 2.60 & 4.00 & 0.93 \\
\hline 32 & Dalbergia latifolia & 400.00 & 0.22 & 4.00 & 0.93 \\
\hline 33 & Asparagus racemosus & 400.00 & 0.22 & 4.00 & 0.93 \\
\hline 34 & Mallotus philippensis & 800.00 & 0.44 & 4.00 & 0.93 \\
\hline 35 & Caryoto urens & 1200.00 & 0.66 & 4.00 & 0.93 \\
\hline 36 & Toona ciliate & 1000.00 & 0.55 & 4.00 & 0.93 \\
\hline 37 & Eleusine indica & 1400.00 & 0.77 & 4.00 & 0.93 \\
\hline 38 & Phyllanthus emblica & 400.00 & 0.22 & 4.00 & 0.93 \\
\hline 39 & Dichanthium sps. & 600.00 & 0.33 & 4.00 & 0.93 \\
\hline 40 & Glochidion velutinum & 1200.00 & 0.66 & 4.00 & 0.93 \\
\hline 41 & Cynodon dactylon & 600.00 & 0.33 & 4.00 & 0.93 \\
\hline 42 & Barleria cristata & 4200.00 & 2.30 & 4.00 & 0.93 \\
\hline 43 & Zizyphus xylopyrus & 400.00 & 0.22 & 2.00 & 0.47 \\
\hline 44 & Thespesia lampas & 600.00 & 0.33 & 2.00 & 0.47 \\
\hline 45 & Pouzolzia zeylanica & 400.00 & 0.22 & 2.00 & 0.47 \\
\hline 46 & Murraya koenigii & 200.00 & 0.11 & 2.00 & 0.47 \\
\hline 47 & Bauhinia vahlii & 200.00 & 0.11 & 2.00 & 0.47 \\
\hline 48 & Galium hirtiflorum & 600.00 & 0.33 & 2.00 & 0.47 \\
\hline 49 & Quercus glauca & 200.00 & 0.11 & 2.00 & 0.47 \\
\hline 50 & Persicaria barbata & 400.00 & 0.22 & 2.00 & 0.47 \\
\hline 51 & Rungia parviflora & 200.00 & 0.11 & 2.00 & 0.47 \\
\hline 52 & Trichilia connaroides & 200.00 & 0.11 & 2.00 & 0.47 \\
\hline 53 & Osyris wightiana & 2000.00 & 1.09 & 2.00 & 0.47 \\
\hline 54 & Dillenia pentagyna & 200.00 & 0.11 & 2.00 & 0.47 \\
\hline 55 & Cassine glauca & 200.00 & 0.11 & 2.00 & 0.47 \\
\hline 56 & Litsea monopetala & 600.00 & 0.33 & 2.00 & 0.47 \\
\hline 57 & Phyllanthus urinaria & 200.00 & 0.11 & 2.00 & 0.47 \\
\hline 58 & Emilia sonchifolia & 200.00 & 0.11 & 2.00 & 0.47 \\
\hline 59 & Mimosa pudica & 400.00 & 0.22 & 2.00 & 0.47 \\
\hline 60 & Trichodesma indicum & 600.00 & 0.33 & 2.00 & 0.47 \\
\hline 61 & Eupatorium adenophorum & 200.00 & 0.11 & 2.00 & 0.47 \\
\hline 62 & Bombax ceiba & 400.00 & 0.22 & 2.00 & 0.47 \\
\hline 63 & Crotalaria prostrata & 1000.00 & 0.55 & 2.00 & 0.47 \\
\hline 64 & Trifolium sp. & 3200.00 & 1.75 & 2.00 & 0.47 \\
\hline 65 & Blumea balsamifera & 1400.00 & 0.77 & 2.00 & 0.47 \\
\hline
\end{tabular}




\section{Vegetation Analysis}

A total of 35 tree species from 19 families were found. The density, relative density, frequency, relative frequency, basal area, relative basal area and IVI value of tree species is presented in Table 6. The study shows higher density of Shorea robusta followed by Anogeissus latifolius. Highest IVI of Shorea robusta shows that it is predominant ecologically important species. Similarly, Anogeissus latifolius comprised the second highest IVI. This indicates that the forest type is predominantly Shorea-Anogeissus type in the study area.

From the stand size classification of observed trees, a high percentage of poles ( $31.51 \%$ ) were found in the sampled plot. Small timber size stand contributes $30.25 \%$ and large saw timber only $16.81 \%$. Study showed that high number of pole-size tree was found and there was poor representation of large saw timber in the study area indicating young forest in the area.

The research showed that Shorea robusta is most dominating species among shrub strata with highest density, and associated common species are Leea crispa and Phoenix humilis. Similarly, Cassia fistula and Anthocephalus chinensis were found to be least common species with density of 8 plants/ha. Highest frequency was both of Shorea robusta and Phoenix humilis i.e.46.0\% followed by Leea crispa with frequency $36 \%$ (Table 7).

A total of 66 species of herb strata was found in the study area with total density of 182600 individual/ha (Table 8). Imperata cylindrica was found to be most common species with highest density (55800 individual/ ha) and other common associated species were Shorea robusta and Pogonatherum crinitum. Likewise, Imperata cylindrica was most frequent herb species found in the study area followed by Shorea robusta.

\section{CONCLUSION}

Households' socio-economic status in Ayodhyapuri Buffer Zone VDC primarily depends on subsistence agricultural system. Land holding size is found to play a vital role in the well beings of local people. The study area is dominated by Brahmin/Chettri and the increasing trend of literacy rate is noted. Efforts have been made to delineate the forest resources in buffer zones under community management but have not achieved much in equal distribution of forest resources to all people in the community. The root problems in the study area are the maximum extraction of fuel wood and fodder from the CF and National Park, and the crop depredation by wildlife. The depredation of the crop by wildlife is one of the major barriers influencing the conservation attitude of local people. The
$\mathrm{CF}$ is rich in biodiversity as revealed by presence of 36 species of tree, 54 species of shrubs and 66 species of herbs. Shorea robusta is the most dominating species in both trees and shrub strata whereas Imperata cylindrica is most frequent herbaceous species. The result shows that the forest is Shorea-Anogeissus type in the study area.

\section{ACKNOWLEDGEMENT}

The authors like to extend their sincere thanks to the Resources Himalaya Foundation, Kathmandu for partial funding to carry out this work. Thanks are also due to the local people of Ayodhyapuri VDC for providing information during questionnaire survey.

\section{REFERENCES}

Basnyat, B. 1999. Tourism and Sustainability: A Case study of Royal Chitwan National Park. B.Sc. dissertation, Institute of Forestry, Pokhara Campus, Pokhara, Nepal.

Budhathoki, P. 2005. Chitwan National Park: A World Heritage Site with Buffer Zone. People \& Protected Areas in South Asia, IUCN/ Resources Himalaya Foundation, Kathmandu, Nepal.

DNPWC/PPP, 2000. Chitwan National Park and Buffer zone Resource Profile. Department of National Parks and Wildlife Conservation/ Park and People Program, Kathmandu, Nepal.

FRSC, 1995. Forest Resources of Chitwan District, 2051. Forest Resource and Survey Centre, Ministry of Forests and Soil Conservation, Kathmandu, Nepal Publication No. 62.

Gautam, K.H., 2006. Forestry, politicians and power-perspectives from Nepal's Forest Policy. Forest Policy and Economics, 8: 175182.

Groom, M.J., Meffe, G.K., Carroll, C.R., 2006. Principles of Conservation Biology. Third Edition. Sinauer Associates, Inc. Publishers, Sunderland, Massachusetts, U.S.A.

K.C., A., 2007. Understanding Biodiversity Conservation and Buffer Zone Vegetation in Manahari Buffer Zone Village Development Committee, Chitwan National Park. M.Sc. dissertation, Central Department of Environmental Science, Tribhuvan University, Kirtipur, Kathmandu.

Nepal, S.K. and Weber, K.E., 1993. Struggle for Existence: Park People Conflict in Royal Chitwan National Park. Studies in Regional Environmental Planning, Division of Human Settlements Development. Asian Institute of Technology, Bangkok, Thailand.

NTNC, 1996. Chitwan National Park after Twenty Years: An Assessment of Values, Threats and Opportunities. National Trust for Nature Conservation, Kathmandu, Nepal.

Oldfield, S., 1988. Buffer Zone Management in Tropical Moist Forest: Case Studies and Guidelines. IUCN, Gland, Switzerland.

Reddy, V.R. 1999. Valuation of Renewable Natural Resources User Perspectives. Economic and Political Weekly, 34 (23), 1435-1441.

Sharma, A., 2000. Wildlife Corridor Management: Analysis of Biodiversity and Socioeconomics in the Buffer Zone of the Royal Chitwan National Park, Nepal. M.Sc. dissertation, Asian Institute of Technology, Thailand.

Zobel, D.B., Jha, P.K., Behan, M. and Yadav, UKR., 1987. A Practical Manual for Ecology. Ratna Book Distributers, Kathmandu. 